\title{
The management of male breast cancer in Nigerians
}

\author{
F.N. Ihekwaba \\ Department of Surgery, University College Hospital, Ibadan, Nigeria
}

\begin{abstract}
Summary: The outcome in 57 male patients with breast cancer has been analysed. Four patients with early disease had simple mastectomy (one case) and radical mastectomy (three cases). All four completed a 12-cycle cyclophosphamide/methotrexate/5-fluorouracil (CMF) course of chemotherapy. Two required orchiectomy after 23 and 38 months, respectively. Two patients were alive at 79 and 63 months. Fifty-three others with advanced disease had simple mastectomy (10 cases), radical mastectomy (38 cases) and no surgery (five cases), but only 33 completed chemotherapy and 11 were submitted to orchiectomy for recurrence after a mean interval of 19 months. Two patients were alive at 63 and 69 months, respectively. The overall survival rate was 7\%. It is considered that in addition to late presentation (mean (SEM): 16.4 (2.1)) months and advanced disease (93\%), ineffectiveness of the CMF regimen in male patients may have contributed to the poor survival rate in these Nigerian patients.
\end{abstract}

Introduction

While the optimal therapy for breast cancer in the female is still incompletely determined, necessitating numerous ongoing clinical trials worldwide, the treatment of males with the disease is even less well understood. ${ }^{1}$ Although problems associated with late presentation, differing pathological classifications and staging criteria and a lack of uniformity in assessing results make treatment results from different centres difficult to compare, all are agreed with the outcome in males with breast cancer is poor. ${ }^{1-3}$

This report details our experience with the management of breast cancer in the Nigerian male at the University College Hospital Ibadan, during the last 20 years.

\section{Patients and methods}

Seventy-nine male patients with breast cancer who presented between April 1971 and April 1990 were reviewed to assess the pathology of the disease and the outcome of treatment. Diagnosis was made from the history and physical examination and confirmed by open biopsy in all cases. Relevant investigations including a full haematological profile, biochemical and enzyme assays of sera, chest radiography and skeletal surveys were obtained. Ultrasonography of the abdomen was obtained in patients seen between 1981 and 1990.

Patients were offered either simple or radical mastectomy with varying degrees of axillary dissection. Orchiectomy was normally withheld until there was evidence of disease progression or local

Correspondence: F.N. Ihekwaba, F.R.C.S.

Accepted: 16 December 1992 recurrence, but it was part of the primary treatment in those who could not be relied upon to submit to it at a later date. Adjuvant combination chemotherapy was administered using cyclophosphamide $100 \mathrm{mg}$ twice daily by mouth (days $1-14$ ), methotrexate $2.5 \mathrm{mg}$ twice daily by mouth (days $1-4$ ) or $50 \mathrm{mg}$ intravenously days 1 and 8 (as available) and 5 -fluorouracil $500 \mathrm{mg}$ intravenously days 1 and 8 , for a planned 12-cycle treatment protocol. A complete course of radiotherapy was not applied in any of the patients. Only 57 patients $(78 \%)$ followed from 1 to 79 months had complete records for analysis. Mean values where appropriate were expressed as mean (SEM).

\section{Results}

\section{Clinical characteristics}

The mean age of the patients was 54 (3.9) years, range 16-80. The mean duration of symptoms was 16.4 (2.1) months, range 4-34. The tumour size varied between 3.5 and $13.5 \mathrm{~cm}$, with a mean of 7 (1.5).

One patient had stage I (T1-2, N0, M0) disease $(2 \%)$, three had stage II (T1-2, N1, M0) (5\%), 33 had stage III (T1-4, N1-3, M0) (58\%) and 20 $(35 \%)$ had stage IV (T1-4, N1-3, M1) disease. Other characteristics of the disease are as shown in Table I.

\section{Histopathology}

The dominant histopathological type $(73 \%)$ was carcinoma, infiltrating ductal and undifferentiated. ${ }^{4}$ Carcinomas of special types - papillary, 
Table I Male breast cancer in Ibadan: some clinical signs in 57 patients

\begin{tabular}{|c|c|c|}
\hline Clinical signs & Patients* & Description \\
\hline \multicolumn{3}{|l|}{ Local disease } \\
\hline Skin involvement & $39 \quad(68)$ & Induration, encrustation, ulceration \\
\hline Nipple involvement & $18 \quad(31)$ & Retraction, ulceration \\
\hline Muscle involvement & 43 (75) & Moderate/firm/fixed \\
\hline \multicolumn{3}{|l|}{ Lymph nodes } \\
\hline Ipsilateral & $51 \quad(91)$ & Mobile - moderate fixity \\
\hline Contralateral & $6 \quad(11)$ & Mobile \\
\hline \multicolumn{3}{|l|}{ Distant disease } \\
\hline Metastases & $10 \quad(18)$ & Pleura, chest wall, liver \\
\hline \multicolumn{3}{|l|}{ Tumour grade } \\
\hline High & $12(50)$ & $24 / 57$ cases \\
\hline Low/intermediate & $12(50)$ & $24 / 3 /$ cases \\
\hline
\end{tabular}

${ }^{*}$ Figures in parentheses are percentages.

colloid, medullary, scirrhous, lobular - accounted for the remainder. In 24 cases in which the malignancy grade was determined, four $(17 \%)$ were low, eight $(33 \%)$ intermediate, while $12(50 \%)$ were high.

\section{Treatment and treatment outcome}

Of the four patients with early disease (stages I and II), one had simple mastectomy while three had radical mastectomy. All four patients completed a 12-cycle course of combination chemotherapy. Two patients developed scar recurrence at 23 and
38 months respectively, from diagnosis requiring excision and bilateral orchiectomy. One of these died at 33 months, while the other defaulted at 63 months, although he was well at his last clinic attendance. The remaining patients were alive at 37 and 79 months, respectively.

Among the 53 patients with advanced disease, five with distant metastases did not receive any local surgery. Only one submitted to primary orchiectomy. All five succumbed within 3 months of presentation.

Of the 48 other patients, 10 had simple mastectomy while 38 had radical mastectomy with satis-
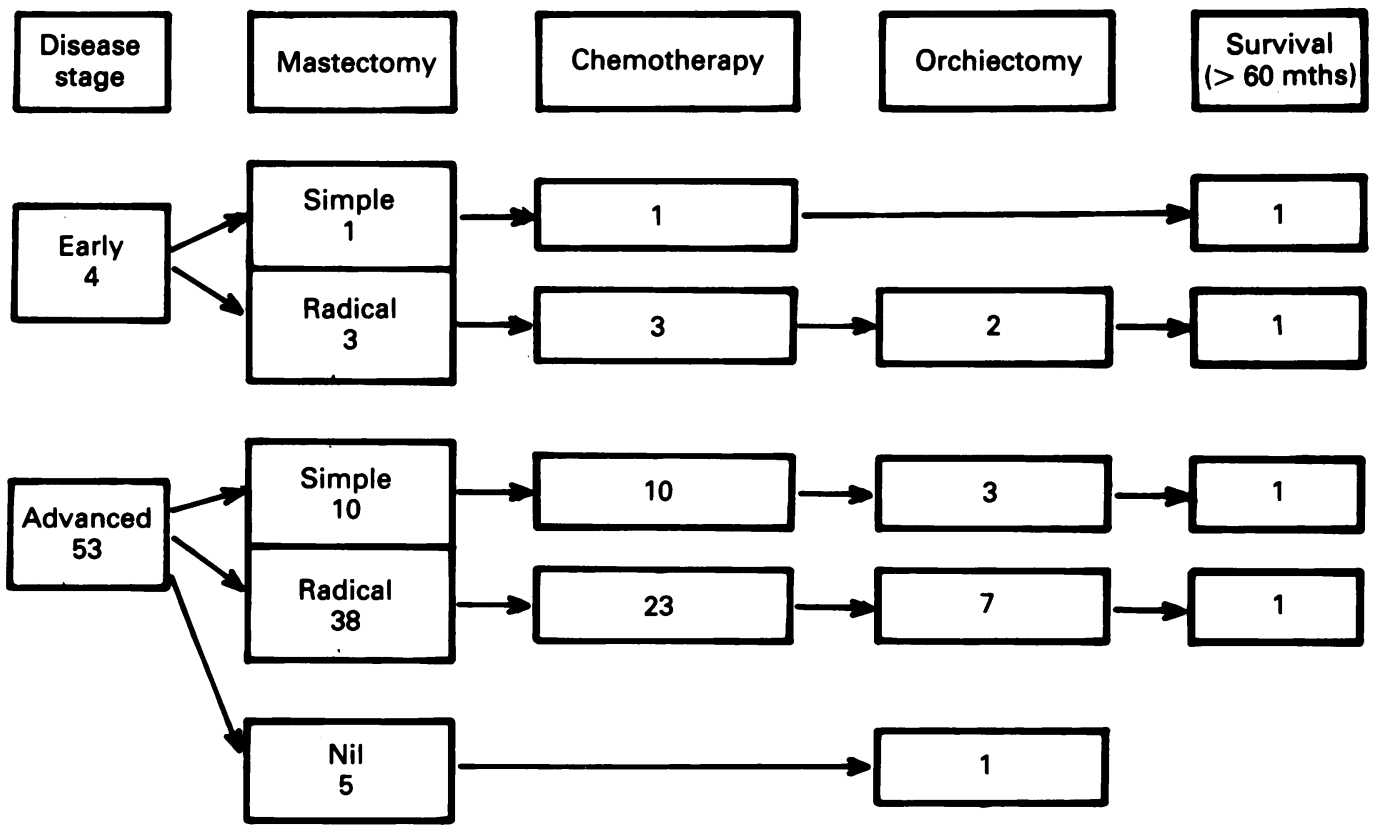

Figure 1 Flow chart for treatment and treatment outcome in 57 patients. 
factory clearance of the axilla in 29 cases $(76 \%)$. All 48 patients were offered a 12-cycle course of chemotherapy. Fifteen patients were observed to deteriorate and either abandoned the course or defaulted at between 4 and 8 months. Among 33 who completed the cyclophosphamide/methotrexate/5-fluorouracil (CMF) programme, 10 submitted to bilateral orchiectomy following evidence of tumour progresssion at between 16 and 39 months, mean 19 months. One patient showed complete response $(10 \%)$ lasting 16 months while two showed partial response of 8 and 11 months duration, respectively, a partial response rate of $20 \%$. Only six patients received tamoxifen for 4-7 months. The response rate for this group was indeterminate because of the limited duration of therapy.

Eight patients among the 33 completing chemotherapy were lost to follow-up at between $13 \frac{1}{2}$ and 23 months from diagnosis. Twenty-one others were known to have died on readmission or at home at between 14 and 69 months (mean $=26$ (3.8)). Only four patients were alive at $28,32,37$ and 63 months, respectively, from diagnosis (Figures 1 and 2).

\section{Discussion}

The optimal treatment for male patients with breast cancer remains controversial., ${ }^{2,3-7}$ While inadequate local experience deriving from a paucity of cases at any one centre may partly be responsible for this, the absence of a reproducible treatment regimen with a high rate of success fuels scepticism when claims for long tumour-free survival are made.

Although radical mastectomy is performed in up to $65 \%$ of patients, ${ }^{1-3,5,8,9}$ it is by no means certain that it affords a greater degree of tumour-free survival than simple mastectomy. ${ }^{6,710}$ Proponents of radical mastectomy have pointed to the sparse breast parenchyma in the male with the tendency to early local spread. It is doubtful if adequate local

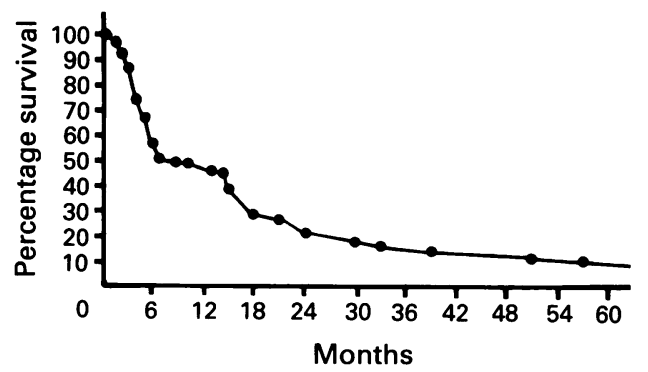

Figure 2 Male breast cancer: percentage survival in 57 patients. clearance is ever achieved in patients with locoregional disease. In this series, radical mastectomy was performed in 32 of 57 patients $(56 \%)$. The difficulty in ensuring adequate axillary clearance was shown by the fact that residual tumour was left behind in one-quarter of cases. Together with 10 others with node positive disease who had simple mastectomy without axillary clearance, it is certain that nearly one-third of patients were left with a substantial tumour burden. This is responsible for the poor results commonly reported from several centres. ${ }^{1,5,11,12}$

Following the introduction of CMF combination as adjuvant therapy in breast cancer ${ }^{13-15}$ there is now widespread acceptance of the efficacy of this drug combination in the treatment of premenopausal women with the disease. Its use in men with the disease, however, is less well clarified. ${ }^{1,11}$ In this study, among 33 with stages III and IV disease completing chemotherapy, 10 harboured recurrent tumours after a mean interval of 19 months. Its probable ineffectiveness in the remainder is suggested by the observation that only five of the 23 patients were alive after 2 years and one after 5 years.

The role of orchiectomy in the control of recurrence is well known.,16-18 The response rate is, however, low (26-45\%). ${ }^{3,17}$ In this study only one patient showed a complete response $(10 \%)$ and two others showed partial responses $(20 \%)$, an overall response rate of approximately $30 \%$. Several? reports ${ }^{5,18,19}$ suggest that the response rate may be increased to $50-80 \%$ in selected patients whose oestrogen and progesterone receptor status are known.

The recent introduction of the anti-oestrogen tamoxifen in the management of these patients may probably alter the survival pattern in the future. Response to tamoxifen is believed to be due to the finding that $80 \%$ of all male breast tumours are oestrogen and progesterone receptor positive., ${ }^{3,17,19}$ On account of the lack of reliable facilities at this centre, no patient in this series received a complete course of radiotherapy.

The crucial role of disease stage in survival is confirmed in this study. Studies among European patients show that those with node negative tumours can expect a 5-year survival greater than $50 \%$ while those with node positive tumours vary between $6 \%$ and $40 \%,{ }^{1,8,9,16}$ In this series, while the only patient with node negative tumour was alive at 79 months, only three among 56 with node positive tumours $(5 \%)$ were alive at 5 years, reflecting the greater number of patients presenting here with disseminated disease. The overall 5-year survival rate from diagnosis for all patients in this series was $7 \%$. 


\section{References}

1. Crichlow, R.W. Carcinoma of the male breast. Surg Gynecol Obstet 1972, 134: 1011-1019.

2. Holleb, A.I., Freeman, E.P. \& Farrow, J.K. Cancer of the male breast. Part I. NY J Med 1968, 68: 544-553.

3. Riberio, C. Male breast cancer. A review of 300 cases from the Christie Hospital and Holt Radium Institute, Manchester. $\mathrm{Br}$ J Cancer 1985, 51: 115-119.

4. Wellings, S.R., Jensen, H.M. \& Maroum, R.G. An atlas of subgross pathology of the human breast with special reference to possible precancerous lesions. J Natl Cancer Inst 1975, 55: 231-273.

5. Donegan, W.L. \& Peres-Mesa, C.M. Carcinoma of the male breast - thirty year review of 28 cases. Arch Surg 1973, 106: 273-279.

6. Spence, R.A.J., Mackenzie, G., Anderson, J.R., Lyons, A.R. \& Bell, N. Long-term survival following cancer of the male breast in Northern Ireland. Report of 81 cases. Cancer 1985, 55: $648-652$.

7. Siddiqui, T., Welner, R., Moreb, J. \& March, R. Cancer of the male breast with prolonged survival. Cancer 1988, 62: 1632-1636.

8. Moss, M.B. Cancer of the male breast. Ann NY Acad Sci 1964, 114: 937-946.

9. Scheike, O. Male breast cancer. Factors influencing prognosis. Br J Cancer 1974, 30: 261-271.

10. Everson, R.B. \& Lippman, M.E. Male breast cancer. In: McGuire, W.L. (ed.). Breast Cancer. Current Topics 3. Plenum, New York, 1979, Vol. 3, 239-267.
11. Satiani, B. \& Powell, R.W. Cancer of the male breast. A 30-year experience. Am Surg 1978, 44: 86-93.

12. Bhagwandeen, S.B. Carcinoma of the male breast in Zambia. East Afr Med J 1972, 49: 89-93.

13. Bonadonna, G., Valagussa, P., Rossi, A. et al. Are surgical adjuvant trials altering the course of breast cancer? Semin Oncol 1978, 5: 450-464.

14. Fisher, B., Slack, N., Katrych, D.L. et al. Ten years follow up results of patients with carcinoma of the breast in a cooperative clinical trials evaluating surgical adjuvant chemotherapy. Surg Gynecol Obstet 1975, 140: 528-534.

15. Breitmeyer, J.B. \& Henderson, I.C. Adjuvant chemotherapy of breast cancer. Surg Clin North Am 1990, 70: 1081-1102.

16. Langlands, A.O., Maclean, N. \& Kerr, G. Cancer of the male breast. A report of 88 cases. Clin Radiol 1976, 27: 21-25.

17. Kantarjian, N., Yap, H.Y., Hortobagyi, G., Buzdar, A. \& Blumenschien, G. Hormonal therapy in metastatic male breast cancer. Arch Intern Med 1983, 143: 237-240.

18. Bezwoda, W.R., Hesdorffer, C., Dansey, R. et al. Breast cancer in men. Clinical features, hormone receptor status and response to therapy. Cancer 1987, 60: 1337-1340.

19. Patel, J.K., Namato, T. \& Dao, T.L. Metastatic breast cancer in males. Assessment of endocrine therapy. Cancer 1984, 53: $1344-1346$. 